\title{
The American Thyroid Association and American Association of Clinical Endocrinologists Hyperthyroidism and Other Causes of Thyrotoxicosis Guidelines: Viewpoints from Japan and Korea
}

\author{
Shunichi Yamashita, ${ }^{1,2}$ Nobuyuki Amino, ${ }^{3,4}$ and Young Kee Shong ${ }^{1.5}$
}

\section{Introduction-Shunichi Yamashita}

T IS WONDERFUL AND VERY INFORMATIVE that this issue of 1 Thyroid is publishing a guidelines paper entitled "Hyperthyroidism and Other Causes of Thyrotoxicosis: Management Guidelines of The American Thyroid Association (ATA) and American Association of Clinical Endocrinologists (AACE)" (1). Of course, the publication is most suitable for the practices of members of the ATA and AACE. For those in other countries, there are areas of agreement but also differences in therapeutic strategy that result from differences in medical background, medical insurance, and the traditions of various medical cultures. Therefore, I asked several representatives of Asia \& Oceania Thyroid Association (AOTA) to comment on the new ATA and AACE guidelines and received two commentaries from Japan and Korea, two of the representative countries in AOTA.

Nearly 10 years ago, three surveys were conducted in which members of the ATA, the European Thyroid Association (ETA), and the Japan Thyroid Association (JTA) were asked about their management of Graves' disease (1). The aim was to determine how expert clinical thyroidologists employed procedures for the diagnosis and differential diagnosis of Graves' disease, and used the three main therapies, antithyroid drugs, radioactive iodine therapy, and surgery. In a summary and analysis of these surveys from these three different regions of the world, both similarities and differences in diagnostic procedures and therapy were noted (2). In general, ATA members used fewer diagnostic tests than did their European or Japanese colleagues. For the index patient, radioiodine was the therapy of choice for $69 \%$ of ATA respondents but only $22 \%$ and $11 \%$ of the ETA and JTA respondents, respectively. In their new management guidelines (1) the ATA and AACE have reconfirmed the choice of their members to preferentially use radioactive iodine treatment for patients with Graves' disease, based on evidence in peerreviewed publications. In contrast, in Japan the first choice for therapy is still antithyroid drugs, now mainly methimazole (MMI).

Regarding diagnostic approaches, the measurement of circulating thyrotropin receptor antibody (TRAb) is used much more frequently in Japan compared with the United States and Korea. It is also considered a good indicator not only for the diagnosis of Graves' disease, but also for monitoring its activity and guiding decisions on decreasing or terminating antithyroid drug therapy.

In his commentary Dr. Amino has briefly reviewed the current situation as reflected by the JTA Guidelines for Management of Graves Hyperthyroidism, first published in Japan in 2006 and appearing as a revision in April 2011. I can only add that the background for the low frequency of choosing radioactive iodine therapy for Graves' patients in Japan is due to a shortage of nuclear therapy facilities and also the negative impact of radiation-induced childhood thyroid cancers after Chernobyl. Fears are now heightened due to the so-called radiophobia following the very serious accident and on-going problems of the Fukushima Dai-ichi nuclear power plant related to the earthquake and tsunami in Japan on March 11, 2011. In addition there is the long-term negative impact and mental trauma from the 1945 atomic bombings of Hiroshima and Nagasaki.

Dr. Young Kee Shong has commented on the very important issue of how to tailor therapeutic strategies for the patients with Graves' disease because they are impacted by dietary intake of relatively high amounts of iodine in certain Asian countries. This is quite different from most of the dietary patterns for iodine in the United States and Canada.

In my opinion there is a continued need to consider the various etiologies of thyrotoxicosis and their pathogenesis in depth as well as how these impact response to treatment. Accumulation of new clinical and basic findings will surely improve and help develop common guidelines for the management of hyperthyroidism to the benefit of patients by facilitating the practices of general clinicians and endocrinologists.

\footnotetext{
${ }^{1}$ Member of the council of the Asia \& Oceania Thyroid Association (AOTA).

${ }^{2}$ Director, Atomic Bomb Disease Institute, Nagasaki University Graduate School of Biomedical Sciences, Nagasaki, Japan.

${ }^{3}$ AOTA advisory member to the World Thyroid Foundation.

${ }^{4}$ Kuma Hospital, Kobe, Japan.

${ }^{5}$ Department of Internal Medicine, Asan Medical Center, University of Ulsan College of Medicine, Seoul, Korea.
} 


\section{Nobuyuki Amino}

The subject matter of the new ATA and AACE Management Guidelines for hyperthyroidism and other causes of thyrotoxicosis (1) are well described for many important issues. Several points differ from the JTA Guidelines for Graves' Hyperthyroidism, however. These were reported in Japanese text in 2006 (3), and revised Japanese Guidelines were published in April 2011. Probably due to the difference in traditional medical background and in medical insurance system, we Japanese endocrinologists prefer to use laboratory tests more frequently in order to detect the side effects of antithyroid drugs earlier, such as agranulocytosis or hepatic injury. Therefore, we recommend routine monitoring of the white blood cell count and differential as well as liver function tests at 2- to 3-week intervals for at least 2 to 3 months after the initiation of antithyroid drugs. In one study it was noted that when $15 \mathrm{mg}$ of MMI daily was used, neutropenia and agranulocytosis were found in $0.255 \%$ and $0.219 \%$ of patients, respectively, and the prevalence of worsening to severe agranulocytosis seemed to be reduced when routine white blood counts were performed (4). Frequent occurrence of hepatic injury was detected by routine liver profile tests, including bilirubin and transaminase (5), likely preventing some cases of hepatic failure. The JTA reported that MMI $15 \mathrm{mg} / \mathrm{d}$ is suitable for mild and moderate Graves' disease, whereas MMI $30 \mathrm{mg} / \mathrm{d}$ is advisable for severe cases. Propylthiouracil is not recommended for initial use because of high frequency of side effects (5).

Gestational thyrotoxicosis is physiological transient hyperthyroidism induced by human chorionic gonadotropin and is found in $3.7 \%$ of normal pregnant women in Japan (6). The most effective method for differentiating it from Graves' disease is the measurement of TRAb by using a rapid and sensitive assay (7). As for postpartum thyrotoxicosis, differentiation between destructive thyrotoxicosis and Graves' hyperthyroidism is essential. Again, a sensitive TRAb assay is very useful, and the results of this assay can be obtained in about 30 minutes. In addition, thyroid blood flow can be measured using ultrasonography and is also effective in distinguishing destructive thyroiditis from Graves' disease. The results of this can be obtained in about 10 minutes (8). We believe that concepts relating to this should be covered in textbooks and guideline recommendations.

In Table 3 of the ATA/AACE guidelines for hyperthyroidism and other causes of thyrotoxicosis (1) we would recommend adding "Familial nonautoimmune hyperthyroidism" as a cause of thyrotoxicosis. At present, this disease is thought to be very rare, just as "resistance to thyroid hormone" was when it was first discovered. If thyrotropin receptor gene mutations are not sought, the diagnosis will not be made. Recently, adult subclinical hyperthyroidism was found to be one form of this disease (9). The entity should be looked for not only in children but also in adults with hyperthyroidism and a diffuse goiter who are TRAb negative. Lastly, we would recommend including "Gonadotropinreleasing hormone analogue-induced thyrotoxicosis" (10) in Table 13 to make it an ideal classification.

\section{Young Kee Shong}

At last, as published in this issue, we have comprehensive management guidelines for the various etiologies of thyro- toxicosis. This is a set of guidelines, based on published evidence, jointly sponsored by the ATA and the AACE (1). The final document was approved by ATA and AACE and officially endorsed by almost all thyroid associations and endocrine societies throughout the world except for AOTA. In most of the AOTA countries the clinical approaches for Graves' disease are very different from those in the United States and prolonged antithyroid drug therapy is usually preferred. Nonetheless, I am sure that the ATA/AACE guidelines will be of great help to those who are learning the causes and management of thyrotoxicosis.

According to the ATA/AACE guidelines (1), Graves' disease is preferentially treated with radioactive iodine. If MMI is chosen as the primary treatment modality, a relatively brief course of the drug is recommended and after a fixed period of 12 to 18 months, it is discontinued and radioactive iodine is administered if the patient relapses. It is well documented that radioiodine therapy will induce permanent hypothyroidism, necessitating permanent levothyroxine therapy as the most cost-effective way to permanently control the hyperthyroidism of Graves' disease. This is certainly true in the United States and western Europe, where medical costs are very high. However, in some countries, where medical costs are not so high, the difference between the various treatment modalities is undoubtedly smaller.

It is well known that increasing dietary iodine intake is associated with an increased incidence of relapse after antithyroid drug therapy for Graves' disease (11-13). Importantly, the efficacy of antithyroid drugs has been evaluated in the literature mostly from moderately iodine-deficient areas, making the interpretation of outcomes very difficult. Notably in Japan and Korea, daily consumption of a variety of seaweed-containing foods is common. Consequently, the amount of iodine in the diet far exceeds the World Health Organization (WHO) allowance and sometimes creates various thyroid problems. Unfortunately, in a recent metaanalysis of the literature that included 26 studies published over the past 18 years (14), only two studies were from areas of excessive iodine intake $(15,16)$. Therefore, there is still not enough data regarding relapse or persistence of active Graves' disease in patients on antithyroid drugs. A recent retrospective study from Asia revealed that prolonged antithyroid therapy was associated with an increased rate of long-term remission (17). Another recent retrospective study from Europe also showed that prolonged antithyroid therapy is clearly associated with higher remission rate (18).

Whether thionamide antithyroid drugs such as MMI have direct immunosuppressive effects is beyond the scope of this commentary, but they likely have more effects than symptomatic treatment with beta-blockers. In the worldwide literature, there is evidence for long-lasting remission in at least $20 \%$ of cases after a standard course of antithyroid drug therapy. This remission rate increases up to $67 \%$ if antithyroid therapy is administered for years (17). If cost is not a consideration, it appears that prolonged antithyroid therapy is relatively safe and is a good option for therapy of Graves' disease, particularly in patients that fear radiation.

TRAb, which is directed against the thyrotropin receptor and stimulates the thyroid-like thyrotropin, is the cause of hyperthyroidism in Graves' disease. TRAb decreases and sometimes disappears during the administration of antithyroid drugs; persistently positive tests for TRAb indicate that 
immediate relapse of hyperthyroidism is likely if the antithyroid drug for treatment of Graves' disease is stopped. Disappearance of TRAb may not indicate long-term remission, however, since in some patients with Graves' disease there may be reappearance of TRAb and recurrence of hyperthyroidism (19). It is a good idea to measure TRAb as well as to conduct thyroid function tests during antithyroid therapy. In patients in whom TRAb disappears and thyroid function normalizes, the antithyroid drug may be withdrawn as soon as disappearance of TRAb is noted (16). In some patients TRAb persists despite years of antithyroid therapy, leading to the need for ongoing treatment with antithyroid drugs. Nevertheless, prolonged antithyroid therapy is a good option if withdrawal of this medication is not possible and the patient's thyroid function remains stable with a minimal dose of antithyroid drug. Some patients might be maintained on a minimal dose of antithyroid drug with or without a small dose of thyroxine for years until spontaneous remission occurs with minimal additional cost. In some patients who have been on antithyroid drugs for many years without evidence of remission, and particularly in those whose thyroid function frequently changes during the prolonged maintenance therapy and those who experience a rise in TRAb activity and aggravation of hyperthyroidism, a good alternative may be high-dose radioiodine therapy with permanent thyroxine replacement likely needed to avoid hypothyroidism. Thyroid surgery would be an alternative to radioactive iodine therapy.

I do not know the exact reason why people in Asian countries have such an irrational and extreme fear of radiation, but thyroidologists in this region agree that patients are usually reluctant to receive radioiodine therapy unless it is for progressive thyroid cancer. For the clinician, it is not easy to persuade a patient to have radioiodine treatment for thyrotoxicosis. It may take long discussions with repeated explanation and sometimes consultation for a second opinion. Patients with Graves' disease may give a very reluctant consent to receive radioiodine therapy only after they have repeated relapses over a span of many years. Although it may seem irrational, it is a social and cultural phenomenon that is not easily changed. In the lecture room or at the bedside I say to my students that radioiodine therapy is the most costeffective method and can be applied to every patient unless there is a specific contraindication, but in the clinic I still cannot persuade my patients to receive radioiodine therapy to treat the hyperthyroidism of Graves' disease with relative ease.

In my opinion Graves' disease is a heterogeneous disorder as far as the response to an antithyroid drug is concerned. In a certain group of patients, generally with small goiter, mild disease, and negative TRAb, a short course of antithyroid therapy is effective and provides long-lasting remission (20). Therefore, we may use prolonged antithyroid drug therapy in selected patients instead of immediate ablative therapy. In others using antithyroid drugs for a relatively short, fixed period to achieve normal thyroid function may be best, with an eventual requirement for ablative treatment, the result of which will be to change the patient's medication from an antithyroid drug to thyroxine. Ideally, well-designed prospective controlled trials are needed to determine the best strategy. This may be very difficult at present because of the high costs and long observation period that would be required for drugs that have been in clinical use for many years.

\section{References}

1. ATA/AACE Taskforce on Hyperthyroidism and Other Causes of Thyrotoxicosis; Bahn RS, Burch HB, Cooper DS, Garber JR, Greenlee MC, Klein I, Laurberg P, McDougall IR, Montori VM, Rivkees SA, Ross DS, Sosa JA, Stan MN 2011 Hyperthyroidism and other causes of thyrotoxicosis: management guidelines of the American Thyroid Association and American Association of Clinical Endocrinologists. Thyroid 21:593-646.

2. Wartofsky L, Glinoer D, Solomon B, Nagataki S, Lagasse R, Nagayama Y, Izumi M 1991 Differences and similarities in the diagnosis and treatment of Graves' disease in Europe, Japan and the United States. Thyroid 1:129-135.

3. Nakamura H 2006 Guideline for treatment of Graves' disease with antithyroid drugs in Japan. Nippon Rinsho 64:2243-2249. In Japanese.

4. Takata K, Kubota S, Fukata S, Kudo T, Nishihara E, Ito M, Amino N, Miyauchi A 2009 Methimazole-induced agranulocytosis in patients with Graves' disease is more frequent with an initial dose of $30 \mathrm{mg}$ daily than with $15 \mathrm{mg}$ daily. Thyroid 19:559-563.

5. Nakamura H, Noh JY, Itoh K, Fukata S, Miyauchi A, Hamada N 2007 Comparison of methimazole and propylthiouracil in patients with hyperthyroidism caused by Graves' disease. J Clin Endocrinol Metab 92:2157-2162.

6. Orito $\mathrm{Y}, \mathrm{Oku} \mathrm{H}, \mathrm{Kubota} \mathrm{S}$, Amino N, Shimogaki K, Hata M, Manki K, Tanaka Y, Sugino S, Ueta M, Kawakita K, Nunotani T, Tatsumi N, Ichihara K, Miyauchi A, Miyake M 2009 Thyroid function in early pregnancy in Japanese healthy women: relation to urinary iodine excretion, emesis, and fetal and child development. J Clin Endocrinol Metab 94:1683-1688.

7. Yoshimura Noh J, Miyazaki N, Ito K, Takeda K, Hiramatsu S, Morita S, Miyauchi A, Murakami T, Inomata K, Noguchi S, Satoh T, Amino N 2008 Evaluation of a new rapid and fully automated electrochemiluminescence immunoassay for thyrotropin receptor autoantibodies. Thyroid 18:1157-1164.

8. Ota H, Amino N, Morita S, Kobayashi K, Kubota S, Fukata S, Kamiyama N, Miyauchi A 2007 Quantitative measurement of thyroid blood flow for differentiation of painless thyroiditis from Graves' disease. Clin Endocrinol (Oxf) 67:41-45.

9. Nishihara E, Chen CR, Higashiyama T, Mizutori-Sasai Y, Ito M, Kubota S, Amino N, Miyauchi A, Rapoport B 2010 Subclinical nonautoimmune hyperthyroidism in a family segregates with a thyrotropin receptor mutation with weakly increased constitutive activity. Thyroid 20:1307-1314.

10. Amino N, Hidaka Y, Takano T, Tatsumi KI, Izumi Y, Nakata Y 2003 Possible induction of Graves' disease and painless thyroiditis by gonadotropin-releasing hormone analogues. Thyroid 13:815-818.

11. Warfofsky L 1973 Low remission rate after therapy for Graves' disease. Possible relation of dietary iodine with antithyroid therapy results. JAMA 26:1083-1088.

12. Roti E, Gardini E, Minelli R, Bianconi L, Salvi M, Gavaruzzi G, Braverman LE 1993 Effects of chronic iodine administration on thyroid status in euthyroid subjects previously treated with antithyroid drugs for Graves' hyperthyroidism. J Clin Endocrinol Metab 76:928-932.

13. Dai WX, Lian XI, Li SH, Li XW 2006 Effect of universal salt iodination on antithyroid drugs. Chin Med J 119:1108-1112.

14. Abraham P, Avenell A, McGreoch SC, Clark LF, Beva JS 2010 Antithyroid drug regimen for treating Graves' hyperthyroidism (Review). Cochrane Database Syst Rev 2010: CD003420. 
15. Hashizume K, Ichikawa K, Sakurai A, Suzuki S, Takeda T, Kobayashi M, Miyamoto T, Arai M, Nagasawa T 1991 Administration of thyroxine in treated Graves' disease. Effects on the level of antibodies to thyroid-stimulating hormone receptors and on the risk of recurrence of hyperthyroidism. N Engl J Med 324:947-953.

16. Cho BY, Shong MH, Yi KH, Lee HK, Koh CS, Min HK 1992 Evaluation of serum basal thyrotropin levels and thyrotropin receptor antibody activities as prognostic markers for discontinuation of antithyroid drug treatment in patients with Graves' disease. Clin Endocrinol 36:585-590.

17. Konishi T, Okamoto Y, Ueda M, Fukuda Y, Harusato I, Tsukamoto Y, Hamada N 2011 Drug discontinuation after treatment with minimum maintenance dose of an antithyroid drug in Graves' disease; a retrospective study on effects of treatment duration with minimum maintenance dose on lasting remission. Endocr J 58:95-100.

18. Mazza E, Carlini M, Flecchia A, Blatto A, Zuccarini O, Gamba S, Beninati S, Messina M 2008 Long-term follow-up of patients with hyperthyroidism due to Graves' disease treated with methimazole. Comparison of usual treatment schedule with drug discontinuation vs continuous treatment with low methimazole doses: a retrospective study. J Endocrinol Invest 31:866-872.

19. Feldt-Rasmussen U, Schleusener H, Carayon P 1994 Metaanalysis evaluation of the impact of thyrotropin receptor antibodies on long term remission after medical therapy of Graves' disease. J Clin Endocrinol Metab 78:98-102.

20. Lauberg P, Buchholtz Hansen PE, Iversen E, Eskjaer Jensen S, Weeke J 1986 Goiter size and outcome of medical treatment of Graves' disease. Acta Endocrinol (Copenh) 111: 39-43.

Address correspondence to: Shunichi Yamashita, M.D. Atomic Bomb Disease Institute Nagasaki University Graduate School of Biomedical Sciences 1-12-4 Sakamoto Nagasaki 852-8523

Japan

E-mail: shun@nagasaki-u.ac.jp 